\title{
ON THE UNIQUENESS OF MARTINGALES WITH CERTAIN PRESCRIBED MARGINALS
}

\author{
MICHAEL R. TEHRANCHI, ${ }^{*}$ University of Cambridge
}

\begin{abstract}
This note contains two main results. (i) (Discrete time) Suppose that $S$ is a martingale whose marginal laws agree with a geometric simple random walk. (In financial terms, let $S$ be a risk-neutral asset price and suppose that the initial option prices agree with the Cox-Ross-Rubinstein binomial tree model.) Then $S$ is a geometric simple random walk. (ii) (Continuous time) Suppose that $S=S_{0} \mathrm{e}^{\sigma X-\sigma^{2}\langle X\rangle / 2}$ is a continuous martingale whose marginal laws agree with a geometric Brownian motion. (In financial terms, let $S$ be a risk-neutral asset price and suppose that the initial option prices agree with the Black-Scholes model with volatility $\sigma>0$.) Then there exists a Brownian motion $W$ such that $X_{t}=W_{t}+o\left(t^{1 / 4+\varepsilon}\right)$ as $t \uparrow \infty$ for any $\varepsilon>0$.
\end{abstract}

Keywords: Fake Brownian motion; binomial tree model; geometric Brownian motion; weak convergence to Brownian motion

2010 Mathematics Subject Classification: Primary 60G42; 60G44; 91B25

\section{Introduction}

Let $S=\left(S_{t}\right)_{t \geq 0}$ be a positive martingale. If we know the marginal laws of the random variables $S_{t}$ for all $t \geq 0$, what can we say about the law of the whole process $S$ ? The contribution of this note is two results which may offer some insight into this question. The first result is in discrete time.

Theorem 1.1. Suppose that $S$ is a positive martingale such that

$$
\mathbb{P}\left(\frac{S_{t}}{S_{0}}=u^{k} d^{t-k}\right)=\left(\begin{array}{l}
t \\
k
\end{array}\right) p^{k}(1-p)^{t-k} \quad \text { for all } 0 \leq k \leq t
$$

for some constants $0<d<1<u$, where

$$
p=\frac{1-d}{u-d} .
$$

Then $\log S$ is a simple random walk with transition probabilities

$$
\mathbb{P}\left(\frac{S_{t}}{S_{t-1}}=u\right)=p=1-\mathbb{P}\left(\frac{S_{t}}{S_{t-1}}=d\right) .
$$

The second result says that the continuous-time analogue of Theorem 1.1 is true in a certain asymptotic sense.

Received 12 June 2012; revision received 28 September 2012.

* Postal address: Statistical Laboratory, Centre for Mathematical Sciences, University of Cambridge, Wilberforce Road, Cambridge CB3 0WB, UK. Email address: m.tehranchi@statslab.cam.ac.uk 
Theorem 1.2. Let $S$ be a positive continuous martingale with respect to a right-continuous complete filtration and such that

$$
\log \left(\frac{S_{t}}{S_{0}}\right) \sim N\left(-\frac{\sigma^{2} t}{2}, \sigma^{2} t\right) \text { for all } t \geq 0
$$

for some constant $\sigma>0$. Let $X$ be the continuous local martingale such that

$$
S_{t}=S_{0} \mathrm{e}^{\sigma X_{t}-\sigma^{2}\langle X\rangle_{t} / 2}
$$

Then there exists a Brownian motion $W$ defined on the same probability space such that

$$
t^{-1 / 4-\varepsilon}\left(X_{t}-W_{t}\right) \rightarrow 0 \text { almost surely (a.s.) as } t \uparrow \infty
$$

for any $\varepsilon>0$. In particular, the law of $X^{n}$ converges weakly to the law of $W$ as $n \uparrow \infty$, where $X_{t}^{n}=n^{-1 / 2} X_{n t}$.

A natural question is whether the local martingale $X$ introduced in Theorem 1.2 must be a Brownian motion itself. Unfortunately, this note does not offer an answer. This question is connected to the existence of so-called fake Brownian motions, but we will defer discussion of this connection to Section 3 below.

The motivation for this study comes from finance. Suppose we model the time- $t$ price of an asset by the random variable $S_{t}$, and we suppose that the process $S=\left(S_{t}\right)_{t \geq 0}$ is a positive martingale under the risk-neutral probability measure. Now consider a European call option written on this asset with maturity date $T \geq 0$ and strike price $K \geq 0$. There would be no arbitrage in the market if the time- 0 price $C_{0}(T, K)$ of this option is given by the formula

$$
C_{0}(T, K)=\mathbb{E}\left[\left(S_{T}-K\right)^{+}\right]
$$

That is to say, the marginal laws of the random variables $S_{t}$ for $t \geq 0$ determine the initial prices of the options.

In practice, however, we do not need to compute option prices. Rather, we can observe the initial stock price $S_{0}$ and a collection of initial option prices $\left\{C_{0}\left(T_{i}, K_{i}\right): i \in I\right\}$. It goes without saying that, in reality, the index set $I$ is finite. However, since the number of observations is large, it is mathematically convenient to pretend that $\left\{\left(T_{i}, K_{i}\right): i \in I\right\}=[0, \infty) \times[0, \infty)$. Since

$$
D_{+} C_{0}(T, K)=-\mathbb{P}\left(S_{T}>K\right),
$$

where $D_{+}$denotes the right-hand derivative in $K$, the collection of option prices determines the marginal laws of the random variables $S_{t}$ for all $t \geq 0$.

From the discussion above, a fundamental modelling problem is to find a martingale $S$ consistent with these observed marginal laws. The first result in this direction is due to Kellerer [14], who showed that there exists a Markovian martingale with a prescribed set of marginal laws so long as those laws have constant mean and increase in the convex order. A concrete formulation of this result is this: we are given a function $C_{0}:[0, \infty) \times[0, \infty) \rightarrow[0, \infty)$ and a number $S_{0}>0$ such that

$$
\begin{array}{ll}
T \mapsto C_{0}(T, K) & \text { is increasing for each } K \geq 0, \\
K \mapsto C_{0}(T, K) & \text { is decreasing and convex for each } T \geq 0
\end{array}
$$


satisfying the boundary conditions

$$
\begin{gathered}
C_{0}(0, K)=\left(S_{0}-K\right)^{+} \quad \text { and } \quad C_{0}(\infty, K) \leq S_{0} \quad \text { for all } K \geq 0, \\
C_{0}(T, 0)=S_{0}, \quad D_{+} C_{0}(T, 0)=-1, \quad \text { and } \quad C_{0}(T, \infty)=0 \quad \text { for all } T \geq 0 .
\end{gathered}
$$

Kellerer showed that there exists a positive martingale $S$ such that $C_{0}(T, K)=\mathbb{E}\left[\left(S_{T}-K\right)^{+}\right]$.

In the financial mathematics literature, Derman and Kani [6] and Dupire [7] considered the problem of inferring asset price dynamics from call prices, in the discrete- and continuous-time settings, respectively. An important observation of Dupire is that, subject to some regularity assumptions on the initial call price surface $C_{0}$, there exists a function $\sigma_{\mathrm{loc}}$ such that the solution $S$ of the stochastic differential equation

$$
\mathrm{d} S_{t}=S_{t} \sigma_{\mathrm{loc}}\left(t, S_{t}\right) \mathrm{d} W_{t}
$$

is consistent with these option prices. (Herein $W$ denotes a Brownian motion.) In particular, the function $\sigma_{\text {loc }}$ is given by the formula

$$
\sigma_{\mathrm{loc}}(T, K)=\left(\frac{2 \partial C_{0}(T, K) / \partial T}{K^{2} \partial^{2} C_{0}(T, K) / \partial K^{2}}\right)^{1 / 2} .
$$

Turning from existence to uniqueness, the alliteratively named paper of Madan and Yor [16] contains a survey of other explicit constructions of Markovian martingales with prescribed marginal laws. It should be noted, however, that apart from the local volatility model of Dupire, the other constructions are necessarily discontinuous martingales. For one of the special cases considered here, that $S$ has the same marginals as geometric Brownian motion, discontinuous constructions based on Skorokhod embeddings have been proposed by $\mathrm{Xu}$ [23].

With this financial context, we can interpret the main results of this note. Recall that the Cox-Ross-Rubinstein [5] binomial tree model for a risk-neutral asset price is a discrete-time martingale $S$ such that $\log S$ is a simple random walk with transition probabilities

$$
\mathbb{P}\left(\frac{S_{t}}{S_{t-1}}=u\right)=\frac{1-d}{u-d}=1-\mathbb{P}\left(\frac{S_{t}}{S_{t-1}}=d\right)
$$

for some constants $0<d<1<u$. The content of Theorem 1.1 is that, perhaps surprisingly, the full dynamics of the Cox-Ross-Rubinstein model (and, hence, the initial prices of pathdependent and American-style options) are fully determined from the initial European call option prices.

The continuous-time version of the binomial tree model is the Black-Scholes model, in which the risk-neutral asset price is modelled as

$$
S_{t}=S_{0} \mathrm{e}^{\sigma W_{t}-\sigma^{2} t / 2}
$$

In this case the call prices are given by the Black-Scholes formula

$$
C_{\mathrm{BS}}\left(S_{0}, T, K, \sigma\right)=S_{0} \Phi\left(\frac{\log \left(S_{0} / K\right)}{\sigma \sqrt{T}}+\frac{\sigma \sqrt{T}}{2}\right)-K \Phi\left(\frac{\log \left(S_{0} / K\right)}{\sigma \sqrt{T}}-\frac{\sigma \sqrt{T}}{2}\right) .
$$

Note that inserting the Black-Scholes formula into Dupire's formula yields $\sigma_{\text {loc }}(T, K)=\sigma$, as it should. The content of Theorem 1.2 is that if the asset price $S$ has continuous trajectories and if the observed option surface is consistent with the Black-Scholes model, then in a certain 
sense the price process $S$, when properly scaled over a very long time horizon, resembles a geometric Brownian motion.

The motivation for studying the question of uniqueness comes from attempts to apply the so-called HJM methodology to call price dynamics. The idea is to treat the dynamics of the whole call price surface as fundamental, rather than derived from the dynamics of the underlying asset price. See the articles of Carmona and Nadtochiy [3], [4], Kallsen and Krühner [13], and Schweizer and Wissel [20], [21] for various partial implementations of this approach.

We now outline one version of this HJM programme, which is very close in spirit (if not in detail) to [3]. The following argument also appears in [22]. Suppose that $F=$ $\left(F_{t}(\tau, m)\right)_{t \geq 0, \tau \geq 0, m \geq 0}$ is a random field evolving according to the stochastic partial differential equation with boundary condition

$$
\begin{aligned}
\mathrm{d} F_{t}(\tau, m)= & \left(\frac{\partial F_{t}}{\partial \tau}-\frac{1}{2} m^{2} \frac{\partial^{2} F_{t}}{\partial m^{2}} \sigma_{t}^{2}+m \frac{\partial B_{t}}{\partial m} \sigma_{t}-B_{t} \sigma_{t}\right) \mathrm{d} t \\
& +B_{t}(\tau, m) \mathrm{d} W_{t} \quad \text { on }(\tau, m) \in(0, \infty) \times[0, \infty), \\
F_{t}(0, m)= & (1-m)^{+} \quad \text { on }(\tau, m) \in\{0\} \times[0, \infty),
\end{aligned}
$$

and suppose that $S$ is a positive local martingale with dynamics

$$
\mathrm{d} S_{t}=S_{t} \sigma_{t} \mathrm{~d} W_{t}
$$

where the random field $B=\left(B_{t}(\tau, m)\right)_{t \geq 0, \tau \geq 0, m \geq 0}$ and process $\sigma=\left(\sigma_{t}\right)_{t \geq 0}$ are given. For each fixed $(T, K)$, define a new process by

$$
C_{t}(T, K)=S_{t} F_{t}\left(T-t, \frac{K}{S_{t}}\right)
$$

Now, by an application of the generalised Itô formula (see Theorem 3.3.1 of [15] for instance) we have

$$
\mathrm{d} C_{t}(T, K)=\left(S_{t} B_{t}\left(T-t, \frac{K}{S_{t}}\right)+S_{t} F_{t}\left(T-t, \frac{K}{S_{t}}\right) \sigma_{t}-K \frac{\partial F_{t}}{\partial m} \sigma_{t}\right) \mathrm{d} W_{t} .
$$

Therefore, by construction, the process $\left(C_{t}(T, K)\right)_{t \in[0, T]}$ is a local martingale such that

$$
C_{T}(T, K)=\left(S_{T}-K\right)^{+}
$$

In particular, the market consisting of a stock with price $S$ and a family $I$ of call options with prices $\left(C_{t}\left(T_{i}, K_{i}\right)\right)_{t \in\left[0, T_{i}\right]}$ for all $i \in I$ is free of arbitrage opportunities. The advantage of this formulation of a market model is that we may take the market observable initial stock price $S_{0}$ and the initial normalized call surface $F_{0}(\tau, m)=C_{0}\left(T, m S_{0}\right) / S_{0}$ as the model input.

To implement this programme, we need only formulate a set of easy-to-check sufficient conditions on the initial prices $S_{0}, F_{0}$ and volatility processes $\sigma, B$ such that (1.1) has a financially meaningful solution. Unfortunately, life is not so simple. Indeed, (1.1) is very poorly behaved. The first hint that there is a problem is that the operator $-\partial^{2} / \partial m^{2}$ does not generate a continuous semigroup with respect to any reasonable function space. Actually, things are even worse. If we insist that the local martingales $S$ and $C(T, K)$ are true martingales, we have the formula

$$
C_{t}(T, K)=\mathbb{E}\left[\left(S_{T}-K\right)^{+} \mid \mathcal{F}_{t}\right]
$$


Durrleman [8] proved that in this case the at-the-money implied volatility tends to the spot volatility as $T \downarrow t$, which in our notation translates into the condition

$$
\sigma_{t}=\sqrt{2 \pi} \lim _{\tau \downarrow 0} \frac{F_{t}(\tau, 1)}{\sqrt{\tau}} .
$$

In particular, the term $-\frac{1}{2} m^{2}\left(\partial^{2} F_{t} / \partial m^{2}\right) \sigma_{t}^{2}$ is actually a cubic nonlinearity! But, from a modelling perspective, we have the important observation that the stock price volatility process $\sigma$ is not a free input to (1.1), but rather it is derived from its solution. The remaining question is, then, how much freedom is there to choose the call price volatility random field $B$ ?

It is hoped that the results of this note help to clarify where the bottleneck in this HJM programme lies. For instance, if $C_{0}(T, K)=C_{\mathrm{BS}}\left(S_{0}, T, K, \sigma_{0}\right)$ for all $(T, K)$ then Corollary 3.1 below says that necessarily

$$
\frac{1}{t} \int_{0}^{t} \sigma_{s}^{2} \mathrm{~d} s \rightarrow \sigma_{0}^{2} \quad \text { a.s. as } t \uparrow \infty .
$$

That is to say, the initial call price surface constrains the possible dynamics of $F$, and in particular, forces the long time average of the squared spot volatility to converge to the initial squared implied volatility.

Remark 1.1. Note that in the original HJM framework, as proposed by Heath et al. [11], the analogous problem does not arise. Recall that if we suppose the random field $\left(f_{t}(\tau)\right)_{t \geq 0, \tau \geq 0}$ evolves according to the HJM-Musiela equation (probably first appearing in this form in [17])

$$
\mathrm{d} f_{t}(\tau)=\left(\frac{\partial f_{t}}{\partial \tau}+b_{t}(\tau) \int_{0}^{\tau} b_{t}(u) \mathrm{d} u\right) \mathrm{d} t+b_{t}(\tau) \mathrm{d} W_{t},
$$

and define $r_{t}=f_{t}(0)$ and $P_{t}(T)=\exp \left[-\int_{0}^{T-t} f_{t}(\tau) \mathrm{d} \tau\right]$, then the process

$$
\left(\exp \left[-\int_{0}^{t} r_{s} \mathrm{~d} s\right] P_{t}(T)\right)_{t \in[0, T]}
$$

is a local martingale for each fixed $T>0$. Interpreting $f$ as the forward rate surface, $r$ as the spot volatility process, and $P$ as the price of a zero-coupon bond, we see that we have a no-arbitrage market model. Furthermore, since the operator $\partial / \partial \tau$ does generate a nice semigroup with respect to almost any function space of interest, we see that only very mild conditions are needed on the random field $b$ to ensure the existence of the financially meaningful solution

$$
f_{t}(\tau)=f_{0}(t+\tau)+\int_{0}^{t} b_{s}(\tau+t-s) \int_{0}^{\tau+t-s} b_{s}(u) \mathrm{d} u \mathrm{~d} s+\int_{0}^{t} b_{s}(\tau+t-s) \mathrm{d} W_{s}
$$

for any initial forward curve $f_{0}$. See, for instance, the lecture notes [9] of Filipovic for a rigorous treatment of this equation.

Now, to carry the analogy further, suppose that the initial bond price curve $P_{0}$ is consistent with a model with constant interest rate $\rho$, so that $P_{0}(\tau)=\mathrm{e}^{-\rho \tau}$ or, equivalently, $f_{0}(\tau)=\rho$ for all $\tau \geq 0$. Unlike the call option case discussed above, nothing can be concluded about the long-time behaviour of the average $t^{-1} \int_{0}^{t} r_{s} \mathrm{~d} s$. For instance, consider the case when $f_{0}(\tau)=\rho$ and $b_{t}(\tau)=\gamma \mathrm{e}^{-\lambda \tau}$. It is straightforward to check the spot rate dynamics are of the VasicekHull-White [12] form

$$
\mathrm{d} r_{t}=\lambda\left(\bar{r}(t)-r_{t}\right) \mathrm{d} t+\gamma \mathrm{d} W_{t},
$$


where the time-varying mean reversion level is $\bar{r}(t)=\rho+\gamma^{2}\left(1-\mathrm{e}^{-2 \lambda t}\right) / 2 \lambda^{2}$. Since we can write $r_{t}$ explicitly as

$$
r_{t}=\rho+\frac{\gamma^{2}}{2 \lambda^{2}}\left(1-\mathrm{e}^{-\lambda t}\right)^{2}+\int_{0}^{t} \gamma \mathrm{e}^{-\lambda(t-s)} \mathrm{d} W_{s},
$$

a routine calculation involving the stochastic Fubini theorem and the Itô isometry shows that

$$
\frac{1}{t} \int_{0}^{t} r_{s} \mathrm{~d} s \rightarrow \rho+\frac{\gamma^{2}}{2 \lambda^{2}} \quad \text { in } L^{2}
$$

In particular, unlike the call option case considered above, the initial forward rate curve does not perfectly predict the long-term average spot interest rate unless $\gamma=0$, i.e. the interest rate dynamics are trivial. This example seems to indicate that the HJM approach to call options differs in a fundamental way from the original HJM approach to interest rate modelling.

The remainder of this short note is organised as follows. In Section 2 we prove Theorem 1.1. In Section 3 we introduce the notion of an $\alpha$-fake Brownian motion. We explore some of the properties of these process, and, in particular, prove Theorem 1.2 above. Finally, in Section 4 we state and prove a few miscellaneous results on $\alpha$-fake Brownian motions which might be useful in either proving that all $\alpha$-fake Brownian motions are true Brownian motions, or else, finding an example of a non-Brownian $\alpha$-fake Brownian motion.

\section{The uniqueness of the Cox-Ross-Rubinstein model}

This section is devoted to the proof of Theorem 1.1. We begin with a lemma.

Lemma 2.1. Let $S$ be a positive martingale. If, for all $t \geq 1$, the random variable $S_{t} / S_{t-1}$ takes values in the set $\{u, d\}$ for some constants $0<d<1<u$, then $\log S$ is a simple random walk with

$$
\mathbb{P}\left(\frac{S_{t}}{S_{t-1}}=u\right)=\frac{1-d}{u-d}=1-\mathbb{P}\left(\frac{S_{t}}{S_{t-1}}=d\right) .
$$

Remark 2.1. Lemma 2.1 is well known. It says that the binomial tree model has exactly one equivalent martingale measure, and so, by the second fundamental theorem of asset pricing, is complete.

Proof of Lemma 2.1. Let $\mathcal{F}$ be the filtration relative to which $S$ is a martingale. Since $S_{t} / S_{t-1}$ can only take two values, the martingale property shows that

$$
S_{t-1}=\mathbb{E}\left[S_{t} \mid \mathcal{F}_{t-1}\right]=S_{t-1} u \mathbb{P}\left(\frac{S_{t}}{S_{t-1}}=u \mid \mathcal{F}_{t-1}\right)+S_{t-1} d \mathbb{P}\left(\frac{S_{t}}{S_{t-1}}=d \mid \mathcal{F}_{t-1}\right) ;
$$

thus,

$$
\mathbb{P}\left(\frac{S_{t}}{S_{t-1}}=u \mid \mathcal{F}_{t-1}\right)=\frac{1-d}{u-d}=1-\mathbb{P}\left(\frac{S_{t}}{S_{t-1}}=d \mid \mathscr{F}_{t-1}\right) .
$$

Since $S_{t} / S_{t-1}$ is manifestly independent of $\mathcal{F}_{t-1}$, we conclude that $\log S$ is a random walk.

Now we are ready for the proof of Theorem 1.1.

Proof of Theorem 1.1. Since $S_{1} / S_{0}$ takes values in $\{u, d\}$, Lemma 2.1 yields

$$
\mathbb{P}\left(S_{1}=u S_{0}\right)=\frac{1-d}{u-d}=1-\mathbb{P}\left(S_{1}=d S_{0}\right) .
$$


Now fix $t \geq 2$ and let

$$
p_{i j}=\mathbb{P}\left(S_{t}=u^{j} d^{t-j} S_{0} \mid S_{t-1}=u^{i} d^{t-1-i} S_{0}\right) \quad \text { for } 0 \leq i \leq t-1,0 \leq j \leq t,
$$

be the one-step transition probabilities. By Lemma 2.1, it is enough to show that

$$
p_{i j}=0 \quad \text { if } j<i \text { or } j>i+1 .
$$

For clarity in the calculations to come, we will use a change of notation:

$$
q=\frac{1-d}{u-1}, \quad r=\frac{u}{d} \quad \Longleftrightarrow \quad u=\frac{r(1+q)}{1+q r}, \quad d=\frac{1+q}{1+q r} .
$$

Now we record the observation that

$$
\sum_{j=0}^{t} p_{i j}=1 \quad \text { for all } 0 \leq i \leq t-1
$$

The martingale property of $S$ yields, in the new notation,

$$
\sum_{j=0}^{t} r^{j} p_{i j}=r^{i} \frac{1+q r}{1+q} \quad \text { for all } 0 \leq i \leq t-1,
$$

and the law of total probability and the prescribed marginal distributions of $S_{t}$ and $S_{t-1}$ yield

$$
\sum_{i=0}^{t}\left(\begin{array}{c}
t-1 \\
i
\end{array}\right) q^{i} p_{i j}=\left(\begin{array}{l}
t \\
j
\end{array}\right) q^{j} \frac{1}{1+q} \quad \text { for all } 0 \leq j \leq t .
$$

We must show that the only nonnegative solution to (2.1), (2.2), and (2.3) is the random walk transition probabilities

$$
\hat{p}_{i j}= \begin{cases}\frac{1}{1+q}=\frac{u-1}{u-d} & \text { if } j=i \\ \frac{q}{1+q}=\frac{1-d}{u-d} & \text { if } j=i+1 \\ 0 & \text { otherwise. }\end{cases}
$$

To this end, introduce a generating function $P$ by the formula

$$
P(x, y)=\sum_{i=0}^{t-1} \sum_{j=0}^{t}\left(\begin{array}{c}
t-1 \\
i
\end{array}\right) p_{i j} x^{i} y^{j}
$$

The functional counterpart to $(2.1)$ is

$$
P(x, 1)=(1+x)^{t-1} \text { for all } x .
$$

Similarly, the counterpart of (2.2) is

$$
P(x, r)=\frac{1+q r}{1+q}(1+x r)^{t-1} \text { for all } x
$$


and the counterpart of $(2.3)$ is

$$
P(q, y)=\frac{1}{1+q}(1+y q)^{t} \text { for all } y .
$$

Now consider the polynomial

$$
\hat{P}(x, y)=\sum_{i=0}^{t-1} \sum_{j=0}^{t}\left(\begin{array}{c}
t-1 \\
i
\end{array}\right) \hat{p}_{i j} x^{i} y^{j}=\frac{1}{q+1}(1+y q)(1+x y)^{t-1}
$$

generated by the geometric random walk transition probabilities $\left(\hat{p}_{i j}\right)_{i, j}$. Of course, since a geometric random walk with these transition probabilities is consistent with the martingale property and the binomial marginals, the polynomial $\hat{P}$ satisfies $(2.4),(2.5)$, and (2.6).

Since $P(x, y)-\hat{P}(x, y)$ is a polynomial of at most degree $t-1$ in $x$ and of degree $t$ in $y$, vanishing when $x=q$ and $y \in\{1, r\}$, we can write

$$
P(x, y)=\hat{P}(x, y)+(q-x)(1-y)(r-y) \sum_{i=0}^{t-2} \sum_{j=0}^{t-2} b_{i, j} x^{i} y^{j} .
$$

Our goal, then, is to show that $b_{i, j}=0$ for all $0 \leq i \leq t-2,0 \leq j \leq t-2$. This will be done by induction.

First, we establish the base case. Matching coefficients of $x^{0} y^{j}$ in (2.7) yields

$$
\begin{aligned}
p_{0, j} & =r b_{0, j}-(1+r) b_{0, j-1}+b_{0, j-2} \quad \text { for all } 2 \leq j \leq t-2, \\
p_{0, t-1} & =-(1+r) b_{0, t-2}+b_{0, t-3}, \\
p_{0, t} & =b_{0, t-2}
\end{aligned}
$$

First we show that the inequality

$$
b_{0, j-1} \geq \frac{r^{t-j}-1}{r^{t-j-1}-1} b_{0, j} \quad \text { for all } 1 \leq j \leq t-2
$$

holds by backward induction. The base case $j=t-2$ is true since

$$
b_{0, t-3}=(1+r) b_{0, t-2}+p_{0, t-1} \geq(1+r) b_{0, t-2} .
$$

Now assuming that

$$
b_{0, J-1} \geq \frac{r^{t-J}-1}{r^{t-J-1}-1} b_{0, J}
$$

holds for some $2 \leq J \leq t-2$, we have

$$
\begin{aligned}
b_{0, J-2} & =(1+r) b_{0, J-1}-r b_{0, J}+p_{0, J} \\
& \geq(1+r) b_{0, J-1}-r b_{0, J} \\
& \geq(1+r) b_{0, J-1}-r \frac{r^{t-J-1}-1}{r^{t-J}-1} b_{0, J-1} \\
& =\frac{r^{t-J+1}-1}{r^{t-J}-1} b_{0, J-1},
\end{aligned}
$$

establishing the inequality for $j=J-1$ and completing the induction. 
It now follows by another induction and the fact that $b_{0, t-2}=p_{0, t} \geq 0$ that

$$
0 \leq \frac{r-1}{r^{t-j-1}-1} b_{0, t-2} \leq b_{0, j} \leq \frac{r^{t-j-1}-1}{r^{t-1}-1} b_{0,0} \quad \text { for all } 0 \leq j \leq t-2 .
$$

Now match the coefficients of $x^{i} y^{0}$ in (2.7):

$$
\left(\begin{array}{c}
t-1 \\
i
\end{array}\right) p_{i, 0}=r q b_{i, 0}-r b_{i-1,0} \quad \text { for all } 1 \leq i \leq t-2, \quad p_{t-1,0}=-r b_{t-2,0}
$$

As before, using the fact that $p_{i, 0} \geq 0$ for all $0 \leq i \leq t-1$ and induction yields

$$
0 \geq q^{t-i-2} b_{t-2,0} \geq b_{i, 0} \geq q^{-i} b_{0,0} \quad \text { for all } 0 \leq i \leq t-2 .
$$

Inequalities (2.8) and (2.9) together imply that $b_{0,0}=0$ and, hence,

$$
b_{i, 0}=b_{0, j}=0 \quad \text { for all } 0 \leq i \leq t-2,0 \leq j \leq t-2 .
$$

Now suppose that

$$
b_{i, h}=b_{h, j}=0 \quad \text { for all } 0 \leq i \leq t-2,0 \leq j \leq t-2 \text {, and } 0 \leq h \leq k-1,
$$

for some $1 \leq k \leq t-3$. As before, we can conclude that

$$
\begin{aligned}
& 0 \leq r b_{k, j}-(1+r) b_{k, j-1}+b_{k, j-2} \quad \text { for all } 2+k \leq j \leq t-2, \\
& 0 \leq-(1+r) b_{k, t-2}+b_{k, t-3} \\
& 0 \leq b_{k, t-2}
\end{aligned}
$$

and

$$
\begin{aligned}
& 0 \leq r q b_{i, k}-r b_{i-1, k} \text { for all } k+1 \leq i \leq t-2 \\
& 0 \leq-r b_{t-2, k}
\end{aligned}
$$

By the same argument as before we see that

$$
b_{i, k}=b_{k, j}=0 \quad \text { for all } 0 \leq i \leq t-2,0 \leq j \leq t-2,
$$

concluding the induction.

Theorem 1.1 has an arithmetic version.

Theorem 2.1. Suppose that $X$ is a martingale such that

$$
\mathbb{P}\left(X_{t}=2 k-t\right)=\left(\begin{array}{l}
t \\
k
\end{array}\right) 2^{-t} \text { for all } 0 \leq k \leq t .
$$

Then $X$ is a simple symmetric random walk.

The proof of the Theorem 1.1 can be adapted to this case. However, we present here a very short and clever argument due to Chris Rogers [19]. 
Proof of Theorem 2.1. First, note that the given law of $X_{t}$ implies that $\mathbb{E}\left[X_{t}\right]=0$ and $\mathbb{E}\left[X_{t}^{2}\right]=t$. Furthermore, since $X$ is a square-integrable martingale, its increments are uncorrelated. In particular, the Pythagorean formula says that

$$
\mathbb{E}\left[X_{t}^{2}\right]=\sum_{s=1}^{t} \mathbb{E}\left[\left(X_{s}-X_{s-1}\right)^{2}\right]
$$

This implies that $\mathbb{E}\left[\left(X_{t}-X_{t-1}\right)^{2}\right]=1$ for all $t \geq 1$. However, since the random variables $X_{t}$ and $X_{t-1}$ take values in the disjoint sets $\{t, t-2, \ldots, 2-t,-t\}$ and $\{t-1, t-3, \ldots, 3-t, 1-t\}$, respectively, we conclude that $\left|X_{t}-X_{t-1}\right| \geq 1$ a.s. But, since $\mathbb{E}\left[\left(X_{t}-X_{t-1}\right)^{2}\right]=1$, we have $X_{t}-X_{t-1} \in\{-1,1\}$ a.s. By the same argument as the proof of Lemma 2.1, the martingale property of $X$ implies that $X$ is a random walk.

\section{Asymptotic uniqueness of the Black-Scholes model}

In this section we will prove Theorem 1.2. However, rather than launching directly into the proof, we begin with a definition.

Definition 3.1. An $\alpha$-fake Brownian motion is a continuous local martingale $X$ with respect to a right-continuous complete filtration such that

$$
X_{t}+\alpha\langle X\rangle_{t} \sim N(\alpha t, t) \text { for all } t \geq 0
$$

To see why it is convenient to offer Definition 3.1, note that if $S=S_{0} \mathrm{e}^{\sigma X-\sigma^{2}\langle X\rangle / 2}$ is a continuous martingale such that

$$
\log \left(\frac{S_{t}}{S_{0}}\right)=\sigma X-\frac{\sigma^{2}\langle X\rangle}{2} \sim N\left(-\frac{\sigma^{2} t}{2}, \sigma^{2} t\right) \quad \text { for all } t \geq 0,
$$

as in the hypothesis of Theorem 1.2, then $X$ is a $-\sigma / 2$-fake Brownian motion.

The notion of fake Brownian motion was introduced recently by Oleszkiewicz [18], corresponding to a 0 -fake Brownian motion in the terminology above. (Actually, Oleszkiewicz also insisted that a fake Brownian motion not be a true Brownian motion, while our definition of $\alpha$-fake Brownian motion does not.) A natural question is whether there are non-Brownian $\alpha$-fake Brownian motions. Hamza and Klebaner [10] gave several constructions for discontinuous martingales with the same marginal laws as Brownian motion, but it seems that Albin [1] was the first to give a construction of a non-Brownian 0-fake Brownian motion. Also, see Oleszkiewicz's paper for several other intuitive constructions, again when $\alpha=0$. Unfortunately, we do not know if there are non-Brownian examples when $\alpha \neq 0$.

We now derive some properties of $\alpha$-fake Brownian motions, which may have some independent interest. Since we are concerned with the case when $\alpha \neq 0$, the following lemma shows that we need only consider $\alpha=1$.

Lemma 3.1. Let $X$ be an $\alpha$-fake Brownian motion with respect to a filtration $\left(\mathcal{F}_{t}\right)_{t \geq 0}$. If $\alpha \neq 0$ then the process $\hat{X}$ given by

$$
\hat{X}_{t}=\alpha X_{t / \alpha^{2}}
$$

is a 1-fake Brownian motion with respect to $\left(\mathcal{F}_{t / \alpha^{2}}\right)_{t \geq 0}$. 
Remark 3.1. Recall that our aim is to prove Theorem 1.2. In particular, the claim that $t^{-1 / 4-\varepsilon}\left(X_{t}-W_{t}\right) \rightarrow 0$ for some Brownian motion $W$ is equivalent to $t^{-1 / 4-\varepsilon}\left(\hat{X}_{t}-\hat{W}_{t}\right) \rightarrow 0$ for the Brownian motion $\hat{W}_{t}=\alpha W_{t / \alpha^{2}}$. In particular, there is no loss assuming that $\alpha=1$.

Proof of Lemma 3.1. Since $X_{s}+\alpha\langle X\rangle_{s} \sim N(\alpha s, s)$ by definition, $\alpha\left(X_{s}+\alpha\langle X\rangle_{s}\right) \sim$ $N\left(\alpha^{2} s, \alpha^{2} s\right)$. The proof is concluded by noting that $\hat{X}_{t}+\langle\hat{X}\rangle_{t}=\alpha X_{s}+\alpha^{2}\langle X\rangle_{s}$, where $t=\alpha^{2} s$.

The following lemma has an elementary proof, but is the key result underlying this study.

Lemma 3.2. Suppose that $X$ is a 1-fake Brownian motion. Then, for all $\lambda<\frac{1}{2}$, we have

$$
\mathbb{E}\left[\mathrm{e}^{\lambda\langle X\rangle_{t}}\right] \leq \mathrm{e}^{\lambda t /(1-2 \lambda)}
$$

for all $t \geq 0$.

Proof. For all $\theta \in \mathbb{R}$, we have

$$
\mathbb{E}\left[\mathrm{e}^{\theta\left(X_{t}+\left\langle X_{t}\right\rangle\right)}\right]=\mathrm{e}^{\left(\theta^{2} / 2+\theta\right) t}
$$

for all $t \geq 0$ since $X_{t} \sim N(t, t)$. Also, since $\mathrm{e}^{\theta X_{t}-\theta^{2}\langle X\rangle_{t} / 2}$ defines a positive local martingale, and, hence, a supermartingale, we have

$$
\mathbb{E}\left[\mathrm{e}^{\theta X_{t}-\theta^{2}\langle X\rangle_{t} / 2}\right] \leq 1
$$

for all $\theta \in \mathbb{R}$ and $t \geq 0$. Hence, by Hölder's inequality,

$$
\begin{aligned}
\mathbb{E}\left[\mathrm{e}^{\lambda\langle X\rangle_{t}}\right] & =\mathbb{E}\left[\left(\mathrm{e}^{2 \lambda\left(X_{t}+\left\langle X_{t}\right\rangle\right) /(1-2 \lambda)}\right)^{(1-2 \lambda) /(2-2 \lambda)}\left(\mathrm{e}^{-2 \lambda X_{t}-(2 \lambda)^{2}\langle X\rangle_{t} / 2}\right)^{1 /(2-2 \lambda)}\right] \\
& \leq \mathbb{E}\left[\mathrm{e}^{2 \lambda\left(X_{t}+\left\langle X_{t}\right\rangle\right) /(1-2 \lambda)}\right]^{(1-2 \lambda) /(2-2 \lambda)} \mathbb{E}\left[\mathrm{e}^{-2 \lambda X_{t}-(2 \lambda)^{2}\langle X\rangle_{t} / 2}\right]^{1 /(2-2 \lambda)} \\
& \leq \mathrm{e}^{\lambda t /(1-2 \lambda)} .
\end{aligned}
$$

Lemma 3.2 yields a useful quantitative estimate.

Lemma 3.3. Suppose that $X$ is a 1-fake Brownian motion. Then, for all $\delta \geq 0$, the inequality

$$
\mathbb{P}\left(\left|\langle X\rangle_{t}-t\right|>\delta\right) \leq 2 \mathrm{e}^{-\delta^{2} / 8(t+\delta)}
$$

holds for all $t \geq 0$.

Proof. By Lemma 3.2 and Markov's inequality, we have, for $0 \leq \lambda<\frac{1}{2}$, the bound

$$
\mathbb{P}\left(\langle X\rangle_{t}-t>\delta\right) \leq \mathbb{E}\left[\mathrm{e}^{\lambda\langle X\rangle_{t}}\right] \mathrm{e}^{-\lambda(t+\delta)} \leq \mathrm{e}^{-\lambda(t+\delta-t /(1-2 \lambda))}=\mathrm{e}^{-(\sqrt{t+\delta}-\sqrt{t})^{2} / 2}
$$

for all $t>0$ and $\delta \geq 0$, where in the equality we set $\lambda=\frac{1}{2}(1-\sqrt{t /(t+\delta)})$. Similarly, we have

$$
\mathbb{P}\left(t-\langle X\rangle_{t}>\delta\right) \leq \mathbb{E}\left[\mathrm{e}^{-\lambda\langle X\rangle_{t}}\right] \mathrm{e}^{\lambda(t-\delta)} \leq \mathrm{e}^{\lambda(t-\delta-t /(1+2 \lambda))}=\mathrm{e}^{-(\sqrt{t}-\sqrt{t-\delta})^{2} / 2}
$$

for all $0 \leq \delta<t$, where now $\lambda=\frac{1}{2}(\sqrt{t /(t-\delta)}-1)>0$. Hence,

$$
\mathbb{P}\left(\left|\langle X\rangle_{t}-t\right|>\delta\right) \leq \mathrm{e}^{-(\sqrt{t+\delta}-\sqrt{t})^{2} / 2}+\mathrm{e}^{-(\sqrt{t}-\sqrt{t-\delta})^{2} / 2} \leq 2 \mathrm{e}^{-\delta^{2} / 8(\delta+t)},
$$

since

$$
\sqrt{t}-\sqrt{t-\delta} \geq \sqrt{t+\delta}-\sqrt{t} \geq \frac{\delta}{2 \sqrt{t+\delta}} .
$$


Corollary 3.1. If $X$ is a 1-fake Brownian motion then

$$
\frac{\langle X\rangle_{t}}{t} \rightarrow 1 \text { a.s. as } t \uparrow \infty .
$$

Proof. Fix a $\delta>0$, and note that Lemma 3.3 says that

$$
\mathbb{P}\left(\left|\frac{\langle X\rangle_{n}}{n}-1\right|>\delta\right) \leq 2 \mathrm{e}^{-n \delta^{2} / 8(1+\delta)} .
$$

Since the right-hand side is summable, the first Borel-Cantelli lemma implies that

$$
\limsup _{n}\left|\frac{\langle X\rangle_{n}}{n}-1\right| \leq \delta \quad \text { a.s. }
$$

and, since $\delta>0$ is arbitrary,

$$
\frac{\langle X\rangle_{n}}{n} \rightarrow 1 \text { a.s. }
$$

Now, for $n \leq t \leq n+1$, we have

$$
\frac{\langle X\rangle_{n}}{n}\left(\frac{n}{n+1}\right) \leq \frac{\langle X\rangle_{t}}{t} \leq \frac{\langle X\rangle_{(n+1)}}{n+1}\left(\frac{n+1}{n}\right) \text { a.s. }
$$

so that

$$
\frac{\langle X\rangle_{t}}{t} \rightarrow 1 \text { a.s. }
$$

as claimed.

The pointwise estimate of Lemma 3.3 can be strengthened to a uniform estimate.

Lemma 3.4. Suppose that $X$ is a 1-fake Brownian motion. Then, for all $\delta \geq 0$, the inequality

$$
\mathbb{P}\left(\max _{0 \leq t \leq T}\left|\langle X\rangle_{t}-t\right|>\delta\right) \leq 2\left(1+\frac{2 T}{\delta}\right) \mathrm{e}^{-\delta^{2} / 32(T+\delta)}
$$

holds for all $T>0$.

Proof. Now note that

$$
\bigcap_{0 \leq k \leq T / \delta+1}\left\{\left|\langle X\rangle_{k \delta}-k \delta\right| \leq \delta\right\} \subseteq\left\{\max _{0 \leq t \leq T}\left|\langle X\rangle_{t}-t\right| \leq 2 \delta\right\},
$$

since if $k \delta \leq t \leq(k+1) \delta$ and $\langle X\rangle_{(k+1) \delta}-(k+1) \delta \leq \delta$ then

$$
\langle X\rangle_{t}-t \leq\langle X\rangle_{(k+1) \delta}-k \delta \leq 2 \delta
$$

and if $\langle X\rangle_{k \delta}-k \delta \geq-\delta$ then

$$
\langle X\rangle_{t}-t \geq\langle X\rangle_{k \delta}-(k+1) \delta \geq-2 \delta .
$$

Therefore, we have the estimate

$$
\begin{aligned}
\mathbb{P}\left(\max _{0 \leq t \leq T}\left|\langle X\rangle_{t}-t\right|>2 \delta\right) & \leq \sum_{0 \leq k \leq T / \delta+1} \mathbb{P}\left(\left|\langle X\rangle_{k \delta}-k \delta\right|>\delta\right) \\
& \leq 2\left(1+\frac{T}{\delta}\right) \mathrm{e}^{-\delta^{2} / 8(T+2 \delta)},
\end{aligned}
$$

where we have used Lemma 3.3 and bounded the sum by the largest term. 
Proof of Theorem 1.2. Let $X$ be a 1-fake Brownian motion. By Corollary 3.1, we have $\langle X\rangle_{t} / t \rightarrow 1$ a.s. and, in particular, $\langle X\rangle_{t} \rightarrow+\infty$ a.s. The Dambis-Dubins-Schwarz theorem yields the existence of a Brownian motion $W$ such that $X_{t}=W_{\langle X\rangle_{t}}$.

Fix $\varepsilon>0$. Our goal is to show that, for all $k>0$, the probabilities

$$
\mathbb{P}\left(n^{-1 / 4-\varepsilon} \max _{n \leq t \leq n+1}\left|X_{t}-W_{t}\right|>k\right)
$$

are summable. Indeed, by the first Borel-Cantelli lemma we would then have

$$
n^{-1 / 4-\varepsilon} \max _{n \leq t \leq n+1}\left|X_{t}-W_{t}\right| \rightarrow 0 \quad \text { a.s. as } n \uparrow \infty,
$$

proving the first claim.

Now note that

$$
\begin{aligned}
& \mathbb{P}\left(n^{-1 / 4-\varepsilon} \max _{n \leq t \leq n+1}\left|X_{t}-W_{t}\right|>k\right) \\
& \quad \leq \mathbb{P}\left(\max _{0 \leq t \leq n+1}\left|\langle X\rangle_{t}-t\right|>n^{1 / 2+\varepsilon}\right) \\
& \quad+\mathbb{P}\left(n^{-1 / 4-\varepsilon} \max _{n \leq t \leq n+1}\left|X_{t}-W_{t}\right|>k, \max _{0 \leq t \leq n+1}\left|\langle X\rangle_{t}-t\right| \leq n^{1 / 2+\varepsilon}\right) .
\end{aligned}
$$

We can use Lemma 3.4 to bound the first term by

$$
\mathbb{P}\left(\max _{0 \leq t \leq n+1}\left|\langle X\rangle_{t}-t\right|>n^{1 / 2+\varepsilon}\right) \leq 2 \exp \left(-n^{2 \varepsilon} \frac{1}{32\left(1+2 n^{-1 / 2+\varepsilon}\right)}\right),
$$

which is summable. The second term is bounded by

$$
\begin{gathered}
\mathbb{P}\left(n^{-1 / 4-\varepsilon} \max _{n \leq t \leq n+1}\left|W_{\langle X\rangle_{t}}-W_{t}\right|>k, \max _{0 \leq t \leq n+1}\left|\langle X\rangle_{t}-t\right| \leq n^{1 / 2+\varepsilon}\right) \\
\leq \mathbb{P}\left(n^{-1 / 4-\varepsilon} \max _{n \leq t \leq n+1,|s-t| \leq n^{1 / 2+\varepsilon}}\left|W_{s}-W_{t}\right|>k\right) .
\end{gathered}
$$

The right-hand side is bounded by

$$
\mathbb{P}\left(\max _{0 \leq t-n \leq 1}\left|W_{t}-W_{n}\right|>\frac{1}{2} n^{1 / 4+\varepsilon} k\right)+\mathbb{P}\left(\max _{|s-n| \leq n^{1 / 2+\varepsilon}+1}\left|W_{s}-W_{n}\right|>\frac{1}{2} n^{1 / 4+\varepsilon} k\right) .
$$

It is clear that the second term dominates the first. By the stationarity of the increments of Brownian motion, the second term is bound by

$$
2 \mathbb{P}\left(\max _{0 \leq s \leq 4 n^{1 / 2+\varepsilon}}\left|W_{s}\right|>\frac{1}{2} n^{1 / 4+\varepsilon} k\right)=2 \mathbb{P}\left(\max _{0 \leq s \leq 1}\left|W_{s}\right|>\frac{1}{4} n^{\varepsilon / 2} k\right)
$$

by Brownian scaling. The right-hand side decays like $\mathrm{e}^{-n^{\varepsilon} C}$ for some constant $C>0$, and, in particular, is summable. The proof that $X_{t}=W_{t}+o\left(t^{1 / 4+\varepsilon}\right)$ a.s. is concluded.

As for the second claim, we first show that the finite-dimensional distributions of $X_{t}^{n}=$ $n^{-1 / 2} X_{n t}$ converge to those of Brownian motion. Let

$$
Y_{t}=(t+1)^{-1 / 2}\left(X_{t}-W_{t}\right)
$$


so that $Y_{t} \rightarrow 0$ a.s. as $t \uparrow \infty$. Then

$$
X_{t}^{n}=W_{t}^{n}+\sqrt{t+\frac{1}{n}} Y_{n t},
$$

where $W_{t}^{n}=n^{-1 / 2} W_{n t}$ is a Brownian motion. For any $t_{1}, \ldots, t_{k}$, we have

$$
\begin{aligned}
\left(X_{t_{1}}^{n}-W_{t_{1}}^{n}, \ldots, X_{t_{k}}^{n}-W_{t_{k}}^{n}\right) & =\left(\sqrt{t_{1}+\frac{1}{n}} Y_{n t_{1}}, \ldots, \sqrt{t_{k}+\frac{1}{n}} Y_{n t_{k}}\right) \\
& \rightarrow 0 \text { a.s., }
\end{aligned}
$$

and of course the random vector $\left(W_{t_{1}}^{n}, \ldots, W_{t_{k}}^{n}\right)$ has the same law as $\left(W_{t_{1}}, \ldots, W_{t_{k}}\right)$. Therefore,

$$
\left(X_{t_{1}}^{n}, \ldots, X_{t_{k}}^{n}\right) \rightarrow\left(W_{t_{1}}, \ldots, W_{t_{k}}\right) \text { in distribution, }
$$

by Theorem 3.1 of [2].

Finally, we will show that the law of the family of processes $\left(X^{n}\right)_{n}$ is tight. Fix a time horizon $T>0$ and $k>0$. Then we have the bound

$$
\begin{aligned}
\mathbb{P}\left(\max _{s, t \in[0, T],|s-t| \leq \delta}\left|X_{t}^{n}-X_{s}^{n}\right|>k\right) \leq & \mathbb{P}\left(\max _{s, t \in[0, T],|s-t| \leq \delta}\left|W_{t}^{n}-W_{s}^{n}\right|>\frac{1}{2} k\right) \\
& +\mathbb{P}\left(\max _{t \in[0, T]}\left(t+\frac{1}{n}\right)^{1 / 2}\left|Y_{n t}\right|>\frac{1}{2} k\right) .
\end{aligned}
$$

We have already shown that the second term on the right vanishes as $n \uparrow \infty$. Hence,

$$
\begin{aligned}
\limsup _{n \uparrow \infty} \mathbb{P}\left(\max _{s, t \in[0, T],|s-t| \leq \delta}\left|X_{t}^{n}-X_{s}^{n}\right|>k\right) & \leq \mathbb{P}\left(\max _{s, t \in[0, T],|s-t| \leq \delta}\left|W_{t}-W_{s}\right|>\frac{1}{2} k\right) \\
& \rightarrow 0
\end{aligned}
$$

as $\delta \downarrow 0$ by the tightness of Wiener measure. The proof is now complete by Theorem 7.5 of [2].

\section{4. $\alpha$-fake miscellany}

Now that we have proven the main results, we conclude with some miscellaneous propositions regarding $\alpha$-fake Brownian motions. The first shows that the case $\alpha=0$ is very different to $\alpha \neq 0$. In particular, in place of Corollary 3.1 above we have the following.

Proposition 4.1. There exists a 0 -fake Brownian motion $X$ such that

$$
\liminf _{t \uparrow \infty} \frac{\langle X\rangle_{t}}{t}=0 \quad \text { a.s. as } t \uparrow \infty .
$$

Proof. This simple construction is due to Oleszkiewicz [18]. Let $B$ and $Y$ be independent Brownian motions, and let $Z$ be an $N(0,1)$ random variable independent of $B$ and $Y$. Define a continuous process $X$ by

$$
X_{t}= \begin{cases}Y_{t} & \text { if } 0 \leq t \leq 1 \\ {\left[Y_{1} \cos \left(B_{\log t}\right)+Z \sin \left(B_{\log t}\right)\right] \sqrt{t}} & \text { if } t>1\end{cases}
$$


It is straightforward to check that $X$ is a martingale in its own filtration. Since $X_{t} \sim N(0, t)$ for all $t \geq 0$ is a 0 -fake Brownian motion. Note that its quadratic variation is given for $t \geq 1$ by the formula

$$
\begin{aligned}
\langle X\rangle_{t} & =1+\int_{1}^{t}\left[-Y_{1} \sin \left(B_{\log s}\right)+Z \cos \left(B_{\log s}\right)\right]^{2} \mathrm{~d} s \\
& =1+R^{2} \int_{0}^{\log t}\left[\sin \left(B_{u}-\theta\right)\right]^{2} \mathrm{e}^{u} \mathrm{~d} u,
\end{aligned}
$$

where $R=\sqrt{Y_{1}^{2}+Z^{2}}$ and $\theta=\tan ^{-1}\left(Z / Y_{1}\right)$. Fix $\varepsilon>0$, and define a sequence of stopping times by $T_{0}=0$, and

$$
\begin{aligned}
T_{2 k-1} & =\inf \left\{u \geq T_{2 k-2}:\left|\sin \left(B_{u}-\theta\right)\right|<\frac{1}{2} \varepsilon\right\} \quad \text { for } k \geq 1, \\
T_{2 k} & =\inf \left\{u \geq T_{2 k-1}:\left|\sin \left(B_{u}-\theta\right)\right|>\varepsilon\right\} \quad \text { for } k \geq 1,
\end{aligned}
$$

and note that

$$
\begin{aligned}
\mathrm{e}^{-T_{2 k}} \int_{0}^{T_{2 k}}\left[\sin \left(B_{u}-\theta\right)\right]^{2} \mathrm{e}^{u} \mathrm{~d} u & \leq \mathrm{e}^{-T_{2 k}} \int_{0}^{T_{2 k-1}} \mathrm{e}^{u} \mathrm{~d} u+\mathrm{e}^{-T_{2 k}} \int_{T_{2 k-1}}^{T_{2 k}} \varepsilon^{2} \mathrm{e}^{u} \mathrm{~d} u \\
& \leq \mathrm{e}^{-\left(T_{2 k}-T_{2 k-1}\right)}+\varepsilon^{2}
\end{aligned}
$$

By the strong Markov property of Brownian motion, the random variables $\left(T_{2 k}-T_{2 k-1}\right)_{k \geq 1}$ are identically distributed and conditionally independent given $\theta$. Since $\mathbb{P}\left(T_{2}-T_{1}>N \mid \theta\right)>0$ a.s. for all $N$, the second Borel-Cantelli lemma shows that, a.s.,

$$
\liminf _{t \uparrow \infty} \frac{\langle X\rangle_{t}}{t} \leq \liminf _{k \uparrow \infty} \mathrm{e}^{-T_{2 k}}\langle X\rangle_{\mathrm{e}^{T_{2 k}}} \leq \varepsilon^{2} R^{2} .
$$

Since $\varepsilon>0$ was arbitrary, we are done.

The remainder of the note is concerned with the $\alpha \neq 0$ case. The next result shows that $\alpha$-Brownian motions have good integrability properties.

Proposition 4.2. Suppose that $X$ is a 1-fake Brownian motion. Then $X$ is a true martingale such that $\mathbb{E}\left[\langle X\rangle_{t}^{p}\right]<\infty$ and $\mathbb{E}\left[\sup _{0 \leq s \leq t}\left|X_{s}\right|^{p}\right]<\infty$ for all $t \geq 0$ and $p \geq 1$.

Proof. The finite exponential moments of $\langle X\rangle_{t}$ from Lemma 3.2 imply that $\mathbb{E}\left[\langle X\rangle_{t}^{p}\right]<\infty$ for all $t \geq 0$ and $p \geq 1$. The result follows from the Burkholder-Davis-Gundy inequality.

In Corollary 3.1 we have proven that $\langle X\rangle_{t} \sim t$ for large $t$. Here we refine this result.

Proposition 4.3. Suppose that $X$ is a 1-fake Brownian motion. Then

$$
\mathbb{E}\left[\langle X\rangle_{t}\right]=t, \quad \operatorname{var}\left(\langle X\rangle_{t}\right) \leq 4 t .
$$

Proof. Since $X_{t}+\langle X\rangle_{t} \sim N(t, t)$ by assumption, we know that

$$
\mathbb{E}\left[X_{t}+\langle X\rangle_{t}\right]=t .
$$

But, by Proposition 4.2 we know that $X$ is not only a local martingale, but a true martingale. Hence, $\mathbb{E}\left[X_{t}\right]=X_{0}=0$, and

$$
\mathbb{E}\left[\langle X\rangle_{t}\right]=t
$$


Now, by Lemma 3.2, the random variable $\langle X\rangle_{t}$ has a moment generating function which is finite on an open neighbourhood of the origin. Hence, we can expand both sides of

$$
\mathbb{E}\left[\mathrm{e}^{\lambda\langle X\rangle_{t}}\right] \leq \mathrm{e}^{\lambda t /(1-2 \lambda)}
$$

in powers of $\lambda$ and compare terms. Since the $\lambda^{0}$ and $\lambda^{1}$ terms agree, we can conclude from the coefficient of $\lambda^{2}$ that

$$
\mathbb{E}\left[\langle X\rangle_{t}^{2}\right] \leq t^{2}+4 t
$$

The next two results lead to sufficient conditions that a 1-fake Brownian motion is a true Brownian motion.

Proposition 4.4. Let $X$ be a 1-fake Brownian motion. Then

$$
\operatorname{var}\left(\langle X\rangle_{t}\right)=-\frac{2}{3} \mathbb{E}\left[X_{t}^{3}\right]
$$

In particular, $X$ is a true Brownian motion if and only if $\mathbb{E}\left[X_{t}^{3}\right] \geq 0$ for all $t \geq 0$.

Remark 4.1. Theorem 4.1 of [22] can be rephrased as follows: if $X$ is a 1-fake Brownian motion such that the conditional distribution of the increments $X_{t}-X_{s}$ given $\mathcal{F}_{s}$ is symmetric for all $0 \leq s \leq t$, then $X$ is a true Brownian motion. Note that Proposition 4.4 above is a generalisation of this result, replacing conditional symmetry of the increments with marginal symmetry of $X_{t}$. In particular, if $X$ is both a 1- and a 0-fake Brownian motion then $X$ is a true Brownian motion. This fact has already been noted in [23].

In fact, by Lemma 3.1, we can rewrite Proposition 4.4 for a general $\alpha$-fake Brownian motion $Y$ as

$$
\mathbb{E}\left[Y_{t}^{3}\right]=-\frac{3}{2} \alpha \operatorname{var}\left(\langle Y\rangle_{t}\right)
$$

Johannes Ruf has observed that the above equality implies that if $Y$ is both an $\alpha_{1}$ - and an $\alpha_{2}$-fake Brownian motion, for $\alpha_{1} \neq \alpha_{2}$, then $Y$ is a true Brownian motion.

Proof of Proposition 4.4. Since $X_{t}+\langle X\rangle_{t} \sim N(t, t)$, we have

$$
\mathbb{E}\left[\left(X_{t}+\langle X\rangle_{t}\right)^{2}\right]=t+t^{2},
$$

and, hence,

$$
\mathbb{E}\left[\langle X\rangle_{t}^{2}\right]=t+t^{2}-2 \mathbb{E}\left[X_{t}\langle X\rangle_{t}\right]-\mathbb{E}\left[X_{t}^{2}\right] .
$$

Since $X$ is a square-integrable martingale with $X_{0}=0$, Lemma 4.3 yields

$$
\mathbb{E}\left[X_{t}^{2}\right]=\mathbb{E}\left[\langle X\rangle_{t}\right]=t,
$$

so that

$$
\operatorname{var}\left(\langle X\rangle_{t}\right)=\mathbb{E}\left[\langle X\rangle_{t}^{2}\right]-\mathbb{E}\left(\langle X\rangle_{t}\right)^{2}=-2 \mathbb{E}\left[X_{t}\langle X\rangle_{t}\right] .
$$

On the other hand, Itô's formula yields the identity

$$
X_{t}^{3}-3 X_{t}\langle X\rangle_{t}=3 \int_{0}^{t}\left(X_{s}^{2}-\langle X\rangle_{s}\right) \mathrm{d} X_{s}
$$


Since the expected quadratic variation of the stochastic integral above can be bounded as

$$
\begin{aligned}
\mathbb{E}\left[\int_{0}^{t}\left(X_{s}^{2}-\langle X\rangle_{s}\right)^{2} \mathrm{~d}\langle X\rangle_{s}\right] & \leq 2 \mathbb{E}\left[\int_{0}^{t}\left(X_{s}^{4}+\langle X\rangle_{s}^{2}\right) \mathrm{d}\langle X\rangle_{s}\right] \\
& \leq 2 \mathbb{E}\left[\langle X\rangle_{t} \sup _{0 \leq s \leq t} X_{s}^{4}+\langle X\rangle_{t}^{3}\right] \\
& \leq 2 \mathbb{E}\left[\langle X\rangle_{t}^{2}\right]^{1 / 2} \mathbb{E}\left[\sup _{0 \leq s \leq t} X_{s}^{8}\right]^{1 / 2}+2 \mathbb{E}\left[\langle X\rangle_{t}^{3}\right],
\end{aligned}
$$

and both expectations appearing on the last line above are finite by Proposition 4.2, the righthand side of (4.1) is a square-integrable martingale, and, in particular,

$$
\mathbb{E}\left[X_{t}^{3}\right]=3 \mathbb{E}\left[X_{t}\langle X\rangle_{t}\right]
$$

Note that if $\mathbb{E}\left[X_{t}^{3}\right]=0$ for all $t \geq 0$ then $\langle X\rangle_{t}=t$ a.s. for all $t \geq 0$, and the conclusion follows from Lévy's characterisation of Brownian motion.

The next proposition gives bounds on the joint moment generating function of $\left(X_{t},\langle X\rangle_{t}\right)$ near the origin.

Proposition 4.5. Suppose that $X$ is a 1 -fake Brownian motion. Fix $t \geq 0$ and $(\theta, \phi)$ such that $2|\phi|<\theta^{2}<1$. Then

$$
\begin{array}{ll}
\mathbb{E}\left[\mathrm{e}^{\theta X_{t}+\phi\langle X\rangle_{t}}\right] \leq \mathrm{e}^{\left(\theta^{2} / 2+\phi\right) t} & \text { if } \theta>0, \\
\mathbb{E}\left[\mathrm{e}^{\theta X_{t}+\phi\langle X\rangle_{t}}\right] \geq \mathrm{e}^{\left(\theta^{2} / 2+\phi\right) t} & \text { if } \theta<0 .
\end{array}
$$

There is equality in either of the above inequalities if and only if $\langle X\rangle_{t}=t$ a.s.

Proof. Note that, since $\theta^{2} / 2<\frac{1}{2}$, we can deduce that $\mathbb{E}\left[\mathrm{e}^{\theta^{2}\langle X\rangle_{t} / 2}\right]<\infty$ by Lemma 3.2. In particular,

$$
\mathbb{E}\left[\mathrm{e}^{\theta X_{t}-\theta^{2}\langle X\rangle_{t} / 2}\right]=1
$$

by Novikov's criterion.

When $\theta>0$, Hölder's inequality yields

$$
\begin{aligned}
\mathbb{E}\left[\mathrm{e}^{\theta X_{t}+\phi\langle X\rangle_{t}}\right] & =\mathbb{E}\left[\left(\mathrm{e}^{\theta\left(X_{t}+\langle X\rangle_{t}\right)}\right)^{\left(\theta^{2}+2 \phi\right) / \theta(2+\theta)}\left(\mathrm{e}^{\theta X_{t}-\theta^{2}\langle X\rangle_{t} / 2}\right)^{2(\theta-\phi) / \theta(2+\theta)}\right] \\
& \leq \mathbb{E}\left[\mathrm{e}^{\theta\left(X_{t}+\left\langle X_{t}\right\rangle\right)}\right]^{\left(\theta^{2}+2 \phi\right) / \theta(2+\theta)} \mathbb{E}\left[\mathrm{e}^{\theta X_{t}-\theta^{2}\langle X\rangle_{t} / 2}\right]^{2(\theta-\phi) / \theta(2+\theta)} \\
& =\mathrm{e}^{\left(\theta^{2} / 2+\phi\right) t} .
\end{aligned}
$$

Similarly, when $\theta<0$, Hölder's inequality once more implies that

$$
\begin{aligned}
1 & =\mathbb{E}\left[\mathrm{e}^{\theta X_{t}-\theta^{2}\langle X\rangle_{t} / 2}\right] \\
& =\mathbb{E}\left[\left(\mathrm{e}^{\theta X_{t}+\phi\langle X\rangle_{t}}\right)^{(2-|\theta|)|\theta| / 2(|\theta|+\phi)}\left(\mathrm{e}^{\theta\left(X_{t}+\langle X\rangle_{t}\right)}\right)^{\left(\theta^{2}+2 \phi\right) / 2(|\theta|+\phi)}\right] \\
& \leq \mathbb{E}\left[\mathrm{e}^{\theta X_{t}+\phi\langle X\rangle_{t}}\right]^{(2-|\theta|)|\theta| / 2(|\theta|+\phi)} \mathbb{E}\left[\mathrm{e}^{\theta\left(X_{t}+\langle X\rangle_{t}\right)}\right]^{\left(\theta^{2}-2 \phi\right) / 2(|\theta|+\phi)} \\
& =\mathbb{E}\left[\mathrm{e}^{\theta X_{t}+\phi\langle X\rangle_{t}}\right]^{(2-|\theta|)|\theta| / 2(|\theta|+\phi)}\left(\mathrm{e}^{-\left(\theta^{2} / 2+\phi\right) t}\right)^{(2-|\theta|)|\theta| / 2(|\theta|+\phi)} .
\end{aligned}
$$

Note that in both cases above, there is equality if and only if $\langle X\rangle_{t}$ is a.s. constant by the criterion for equality in Hölder's inequality. But, Lemma 4.3 says that $\mathbb{E}\left[\langle X\rangle_{t}\right]=t$, and, hence, $\langle X\rangle_{t}$ is a.s. constant if and only if $\langle X\rangle_{t}=t$ a.s. 
Remark 4.2. The same argument as in Proposition 4.5 can be used to bound the moment generating function of $X$ further from the origin. For instance, the inequality

$$
\begin{aligned}
\mathbb{E}\left[\mathrm{e}^{\theta X_{t}}\right] & =\mathbb{E}\left[\left(\mathrm{e}^{\theta\left(X_{t}+\left\langle X_{t}\right\rangle\right)}\right)^{\theta /(2+\theta)}\left(\mathrm{e}^{\theta X_{t}-\theta^{2}\langle X\rangle_{t} / 2}\right)^{2 /(2+\theta)}\right] \\
& \leq \mathbb{E}\left[\mathrm{e}^{\theta\left(X_{t}+\left\langle X_{t}\right\rangle\right)}\right]^{\theta /(2+\theta)} \mathbb{E}\left[\mathrm{e}^{\theta X_{t}-\theta^{2}\langle X\rangle_{t} / 2}\right]^{2 /(2+\theta)} \\
& \leq \mathrm{e}^{\theta^{2} t / 2}
\end{aligned}
$$

holds for all $\theta \geq 0$. In particular, for all $x \geq 0$ and $\theta \geq 0$, we have

$$
\mathbb{P}\left(X_{t}>x\right) \leq \mathbb{E}\left[\mathrm{e}^{\theta X_{t}}\right] \mathrm{e}^{-\theta x} \leq \mathrm{e}^{\theta^{2} t / 2-\theta x}=\mathrm{e}^{-x^{2} / 2 t},
$$

where we have let $\theta=x / t$ in the equality.

Proposition 4.6. Let $X$ be a 1-fake Brownian motion with respect to a probability measure $\mathbb{P}$. Define a locally equivalent measure $\mathbb{Q}$ by

$$
\frac{\mathrm{d} \mathbb{Q}}{\mathrm{d} \mathbb{P}} \mid \mathcal{F}_{t}=\mathrm{e}^{-2 X_{t}-2\langle X\rangle_{t}} .
$$

Then $\mathbb{Q}$ is a probability measure under which the process

$$
Y_{t}=X_{t}+2\langle X\rangle_{t}
$$

is a-1-fake Brownian motion.

Proof. Let

$$
M_{t}=\mathrm{e}^{-2 X_{t}-2\langle X\rangle_{t}} .
$$

Since $X_{t}+\langle X\rangle_{t} \sim N(t, t)$ under $\mathbb{P}$, we have

$$
\mathbb{E}^{\mathbb{P}}\left[M_{t}\right]=\mathbb{E}\left[\mathrm{e}^{-2\left(X_{t}+\langle X\rangle_{t}\right)}\right]=1,
$$

so that $\mathbb{Q}$ is a probability measure. Also, since

$$
M_{t}=\mathrm{e}^{-2 X_{t}-(-2)^{2}\langle X\rangle_{t} / 2},
$$

Girsanov's theorem implies that $Y=X+2\langle X\rangle$ is a $\mathbb{Q}$-local martingale. Finally, since, for all $\theta \in \mathbb{R}$, we have the calculation

$$
\mathbb{E}^{\mathbb{Q}}\left[\mathrm{e}^{\theta\left(Y_{t}-\langle Y\rangle_{t}\right)}\right]=\mathbb{E}^{\mathbb{P}}\left[\mathrm{e}^{(\theta-2)\left(X_{t}+\langle X\rangle_{t}\right)}\right]=\mathrm{e}^{\left(\theta^{2} / 2-\theta\right) t},
$$

we see that $Y_{t}-\langle Y\rangle_{t} \sim N(-t, t)$ under $\mathbb{Q}$ for all $t \geq 0$, concluding the proof.

\section{Acknowledgements}

I would like to thank Chris Rogers, Jan Oblój, Sergey Nadtochiy, and Yang Lu for interesting discussions of the ideas which lead to this note. In particular, thanks to Yang for bringing to my attention references [1] and [18]. This work was presented at the UT-Austin Portugal Workshop on Mathematical Finance in Lisbon, July 2012. I would like to thank the participants for their feedback. Thanks also to the anonymous referee for a thorough reading of, and a detailed list of comments on, a preliminary version of this note. 


\section{References}

[1] Albin, J. M. P. (2008). A continuous non-Brownian motion martingale with Brownian motion marginal distributions. Statist. Prob. Lett. 78, 682-686.

[2] Billingsley, P. (1999). Convergence of Probability Measures, 2nd end. John Wiley, New York.

[3] Carmona, R. and Nadtochiy, S. (2009). Local volatility dynamic models. Finance Stoch. 13, 1-48.

[4] Carmona, R. and Nadtochiy, S. (2012). Tangent Lévy market models. Finance Stoch. 16, 63-104.

[5] Cox, J. C., Ross, S. A. And Rubinstein, M. (1979). Option pricing: a simplified approach. J. Financial Econom. 7, 229-263.

[6] Derman, E. and Kani, I. (1994). The volatility smile and its implied tree. Goldman Sachs Quantitative Strategies Research Notes.

[7] Dupire, B. (1994). Pricing with a smile. Risk 7, 32-39.

[8] Durrleman, V. (2008). Convergence of at-the-money implied volatilities to the spot volatility. J. Appl. Prob. 45, 542-550.

[9] Filipović, D. (2001). Consistency Problems for Heath-Jarrow-Morton Interest Rate Models (Lecture Notes Math. 1760). Springer, Berlin.

[10] Hamza, K. and Klebaner, F. C. (2007). A family of non-Gaussian martingales with Gaussian marginals. J. Appl. Math. Stoch. Anal. 2007, article ID 92723, 19 pp.

[11] Heath, D., Jarrow, R. and Morton, A. (1992). Bond pricing and the term structure of interest rates: a new methodology for contingent claims valuation. Econometrica $\mathbf{6 0 , 7 7 - 1 0 5 . ~}$

[12] Hull, J. C. and White, A. (1990). Pricing interest-rate-derivative securities. Rev. Financial Studies 3, 573-592.

[13] Kallsen, J. And KrüHner, P. (2010). On a Heath-Jarrow-Morton approach for stock options. Preprint.

[14] Kellerer, H. G. (1972). Markov-Komposition und eine Anwendung auf Martingale. Math. Ann. 198, 99-122.

[15] Kunita, H. (1997). Stochastic Flows and Stochastic Differential Equations. Cambridge University Press.

[16] Madan, D. B. ANd YoR, M. (2002). Making Markov martingales meet marginals: with explicit constructions. Bernoulli 8, 509-536.

[17] Musiela, M. (1993). Stochastic PDEs and term structure models. In Journées Internationales de Finance, IGR-AFFI, La Baule.

[18] Oleszkiewicz, K. (2008). On fake Brownian motions. Statist. Prob. Lett. 78, 1251-1254.

[19] Rogers, L. C. G. (2009). A martingale with binomial marginals is a simple random walk. Personal communication.

[20] SCHWEIZER, M. AND Wissel, J. (2006). Term structures of implied volatilities: absence of arbitrage and existence results. Math. Finance 18, 77-114.

[21] Schweizer, M. AND Wissel, J. (2008). Arbitrage-free market models for option prices: the multi-strike case. Finance Stoch. 12, 469-505.

[22] Tehranchi, M. R. (2009). Symmetric martingales and symmetric smiles. Stoch. Process. Appl. 119, 3785-3797.

[23] Xu, X. (2011). Fake geometric Brownian motion and its option pricing. MSc Thesis, Oxford University. 